\title{
Electromagnetic moments of the bound system of charged particles
}

\author{
Albert Wienczek, ${ }^{1}$ Mariusz Puchalski, ${ }^{2}$ and Krzysztof Pachucki ${ }^{1}$ \\ ${ }^{1}$ Faculty of Physics, University of Warsaw, Hoża 69, 00-681 Warsaw, Poland \\ ${ }^{2}$ Faculty of Chemistry, Adam Mickiewicz University, Grunwaldzka 6, 60-780 Poznań, Poland
}

\begin{abstract}
We consider a bound system of particles interacting via electromagnetic forces in an external electromagnetic field, including leading relativistic corrections. Each particle has a definite mass, charge, spin, and charge radius. We introduce suitable canonical transformations and a perturbation scheme to obtain a Hamiltonian which describes the global dynamics of the system. This enables the derivation of formulas for various electromagnetic moments, such as the magnetic dipole moment, the mean square charge radius, and the electric dipole polarizability.

PACS numbers: 32.10.Dk, 31.15.-p, 31.30.js
\end{abstract}

\section{INTRODUCTION}

Let us consider a system of particles, like an atom, an ion, a molecule, or a nucleus, which forms a bound state. We are interested in the motion and global properties of this system in an external electromagnetic field. When relativistic corrections are included, the center of mass cannot be separated from internal degrees of freedom and this may cause appearance of additional corrections to electromagnetic moments. A typical problem is the magnetic moment of the system, for which the first correct description was presented by Hegstrom in [1]. Here we aim to present an approach, on the basis of previously obtained results, which allows one to obtain various electromagnetic moments including relativistic corrections, e.g. the charge radius and the electric dipole polarizability. Although we consider electromagnetic systems herein, the approach can be extended to a nonrelativistic system of strongly interacting particles, such as nuclei.

\section{PERTURBATIVE APPROACH TO SEPARATION OF THE CENTER OF MASS MOTION}

We assume that the Hamiltonian $H$ for a system of particles can be decomposed as

$$
H=H_{\mathrm{S}}+H_{\Pi}+\delta H,
$$

where $H_{\mathrm{S}}$ is the Hamiltonian that involves only internal degrees of freedom. $H_{\Pi}$ is the Hamiltonian for the global dynamics of the system, which involves center of mass and electromagnetic moments. $\delta H$ is the remainder, which couples internal degrees of freedom to global motion. We will assume that the binding energy is much larger than the characteristic scale of energy in $\delta H$, so it makes sense to speak about a bound system. We will also assume that global motion is nonrelativistic. We aim to find an effective equation for the global motion that accounts for the coupling to internal degrees of freedom. The Schrödinger equation for the total system is

$$
i \frac{\partial \psi}{\partial t}=\left(H-\mathcal{E}_{\mathrm{S}}\right) \psi,
$$

where the leading factor that comes from the binding energy $\mathcal{E}_{\mathrm{S}}$ was subtracted from the time dependence. The Hamilto- nians $H_{\mathrm{S}}$ and $H_{\Pi}$ involve different degrees of freedom of the system, so they commute

$$
\left[H_{\mathrm{S}}, H_{\Pi}\right]=0,
$$

and it allows one to decompose the global wave function as follows

$$
\psi=\psi_{\mathrm{S}} \psi_{\Pi}+\delta \psi
$$

where $\psi_{\mathrm{S}}$ is the wave function of the ground state of internal Hamiltonian $H_{\mathrm{S}}$ with corresponding energy $\mathcal{E}_{\mathrm{S}}$, namely

$$
H_{\mathrm{S}} \psi_{\mathrm{S}}=\mathcal{E}_{\mathrm{S}} \psi_{\mathrm{S}} .
$$

$\psi_{\Pi}$ in Eq. (4) is the wave function describing the global dynamics of the system, so it depends only on global degrees of freedom. The last term in Eq. (4), $\delta \psi$, is a small correction that depends on all the variables, and results from the coupling of internal and external degrees of freedom. We will assume that

$$
\begin{aligned}
\langle\psi \mid \psi\rangle & =1=\left\langle\psi_{\mathrm{S}} \mid \psi_{\mathrm{S}}\right\rangle_{\mathrm{S}}, \\
\left\langle\psi_{\mathrm{S}} \mid \delta \psi\right\rangle_{\mathrm{S}} & =0 \\
\left\langle\psi_{\mathrm{S}}|\delta H| \psi_{\mathrm{S}}\right\rangle_{\mathrm{S}} & =0
\end{aligned}
$$

where $\langle\ldots\rangle_{S}$ denotes the scalar product on internal degrees of freedom only. If the last condition (8) is not satisfied, one can always redefine $H_{\Pi}$ to include $\left\langle\psi_{\mathrm{S}}|\delta H| \psi_{\mathrm{S}}\right\rangle_{S}$, and subtract this expectation value from $\delta H$. As a result, this condition does not reduce generality. Let us now project the Schrödinger equation (2) into $\psi_{\mathrm{S}}$, then using assumptions in (6) - (8) one obtains

$$
i \frac{\partial \psi_{\Pi}}{\partial t}=H_{\Pi} \psi_{\Pi}+\left\langle\psi_{\mathrm{S}}|\delta H| \delta \psi\right\rangle_{\mathrm{S}} .
$$

Eq. (2) with the wave function (4) can be rewritten in the form

$$
\begin{aligned}
\left(\mathcal{E}_{\mathrm{S}}-H_{\mathrm{S}}-H_{\Pi}+i \frac{\partial}{\partial t}\right) \delta \psi= & \psi_{S}\left(H_{\Pi}-i \frac{\partial}{\partial t}\right) \psi_{\Pi} \\
& +\delta H\left(\psi_{\mathrm{S}} \psi_{\Pi}+\delta \psi\right)(10)
\end{aligned}
$$

and formally

$$
\delta \psi=\frac{1}{\left[\left(\mathcal{E}_{\mathrm{S}}-H_{\mathrm{S}}\right)^{\prime}-H_{\Pi}+i \partial_{t}\right]} \delta H\left(\psi_{\mathrm{S}} \psi_{\Pi}+\delta \psi\right),
$$


where prime denotes exclusion of the ground state from the resolvent, which is defined by the following series:

$\delta \psi=\left[\frac{1}{\left(\mathcal{E}_{\mathrm{S}}-H_{\mathrm{S}}\right)^{\prime}}+\frac{H_{\Pi}-i \partial_{t}}{\left(\mathcal{E}_{\mathrm{S}}-H_{\mathrm{S}}\right)^{\prime 2}}+\ldots\right] \delta H\left(\psi_{\mathrm{S}} \psi_{\Pi}+\delta \psi\right)$.

$\delta H$ is assumed to be a small correction, so we neglect $\delta \psi$ on the right hand side of Eq. (12)

$$
\delta \psi=\frac{1}{\left[\left(\mathcal{E}_{\mathrm{S}}-H_{\mathrm{S}}\right)^{\prime}-H_{\Pi}+i \partial_{t}\right]} \delta H \psi_{\mathrm{S}} \psi_{\Pi} .
$$

$H_{\Pi}$ and the characteristic time scale of $\delta H$ is much smaller then the excitation energy of $H_{S}$, so we use Eq. (12) and neglect the higher order terms

$$
\delta \psi=\frac{1}{\left(\mathcal{E}_{\mathrm{S}}-H_{\mathrm{S}}\right)^{\prime}} \delta H \psi_{\mathrm{S}} \psi_{\Pi}+\ldots
$$

Finally, the equation for $\psi_{\Pi}$ becomes

$$
\begin{aligned}
i \frac{\partial \psi_{\Pi}}{\partial t} & =H_{\mathrm{eff}} \psi_{\Pi} \\
H_{\mathrm{eff}} & =H_{\Pi}+\left\langle\psi_{\mathrm{S}}\left|\delta H \frac{1}{\left(\mathcal{E}_{\mathrm{S}}-H_{\mathrm{S}}\right)^{\prime}} \delta H\right| \psi_{\mathrm{S}}\right\rangle_{S}+\ldots
\end{aligned}
$$

which can be rewriten in the more convenient form

$H_{\mathrm{eff}}=\left\langle\psi_{S}\left|H-\mathcal{E}_{S}\right| \psi_{S}\right\rangle_{S}+\left\langle\psi_{\mathrm{S}}\left|H \frac{1}{\left(\mathcal{E}_{\mathrm{S}}-H_{\mathrm{S}}\right)^{\prime}} H\right| \psi_{\mathrm{S}}\right\rangle_{S}+\ldots$
In the actual calculations we perform additional canonical transformations to avoid the higher order terms denoted by dots in the above.

\section{HAMILTONIAN FOR THE COMPOUND SYSTEM}

We consider now a system of $N$ charged particles placed in the external electromagnetic field, including the leading relativistic corrections. We assume that the magnetic field is homogenous, and for the electric field we keep the first derivatives to account for the charge radius. We would like to separate the center-of-mass motion and obtain general formulae for electromagnetic moments, such as the magnetic dipole moment $\mu$, the charge radius, and the electric dipole polarizabiliy. Our approach is based on Refs. [2, 3], where relativistic effects are included perturbatively, while the wave function is nonrelativistic and includes the spin. The initial Hamiltonian is a sum of one-particle terms $H_{a}$ and two-particle interactions $H_{a b}$ including relativistic corrections [4, 5] (using natural units $\hbar=c=1$ )

$$
H=\sum_{a} H_{a}+\sum_{a>b, b} H_{a b},
$$

with

$$
\begin{aligned}
H_{a}= & \frac{\vec{\pi}_{a}^{2}}{2 m_{a}}+e_{a} A_{a}^{0}-\frac{e_{a}}{2 m_{a}} g_{a} \vec{s}_{a} \cdot \vec{B}_{a}-\frac{e_{a}}{4 m_{a}^{2}}\left(g_{a}-1\right) \vec{s}_{a} \cdot\left(\vec{E}_{a} \times \vec{\pi}_{a}-\vec{\pi}_{a} \times \vec{E}_{a}\right) \\
& -\frac{\vec{\pi}_{a}^{4}}{8 m_{a}^{3}}-\frac{e_{a}}{6} r_{E a}^{2} \nabla \cdot \vec{E}_{a}+\frac{e_{a}}{8 m_{a}^{3}}\left[4 \vec{\pi}_{a}^{2} \vec{s}_{a} \cdot \vec{B}_{a}+\left(g_{a}-2\right)\left\{\vec{\pi}_{a} \cdot \vec{B}_{a}, \vec{\pi}_{a} \cdot \vec{s}_{a}\right\}\right], \\
H_{a b}= & \frac{e_{a} e_{b}}{4 \pi}\left\{\frac{1}{r_{a b}}-\frac{1}{2 m_{a} m_{b}} \pi_{a}^{i}\left(\frac{\delta^{i j}}{r_{a b}}+\frac{r_{a b}^{i} r_{a b}^{j}}{r_{a b}^{3}}\right) \pi_{b}^{j}-\frac{2 \pi}{3}\left(r_{E a}^{2}+r_{E b}^{2}\right) \delta^{3}\left(r_{a b}\right)\right. \\
& +\frac{1}{2 r_{a b}^{3}}\left[\frac{g_{a}}{m_{a} m_{b}} \vec{s}_{a} \cdot \vec{r}_{a b} \times \vec{\pi}_{b}-\frac{g_{b}}{m_{a} m_{b}} \vec{s}_{b} \cdot \vec{r}_{a b} \times \vec{\pi}_{a}+\frac{\left(g_{b}-1\right)}{m_{b}^{2}} \vec{s}_{b} \cdot \vec{r}_{a b} \times \vec{\pi}_{b}\right. \\
& \left.\left.-\frac{\left(g_{a}-1\right)}{m_{a}^{2}} \vec{s}_{a} \cdot \vec{r}_{a b} \times \vec{\pi}_{a}\right]-\frac{2 \pi g_{a} g_{b}}{3 m_{a} m_{b}} \delta^{3}\left(r_{a b}\right) \vec{s}_{a} \cdot \vec{s}_{b}+\frac{g_{a} g_{b}}{4 m_{a} m_{b}} \frac{s_{a}^{i} s_{b}^{j}}{r_{a b}^{3}}\left(\delta^{i j}-\frac{3 r_{a b}^{i} r_{a b}^{j}}{r_{a b}^{2}}\right)\right\},
\end{aligned}
$$

where $\vec{\pi}=\vec{p}-e A(\vec{r}) . r_{E a}^{2}$ is the mean square charge radius of a particle $a$ and it includes for convenience the Darwin term, so for the point $s=1 / 2$ particle $r_{E}^{2}=3 /\left(4 m^{2}\right)$. We now introduce global variables, the center of mass $\vec{R}$, and the total momentum $\vec{\Pi}$

$$
\begin{aligned}
\vec{R} & =\sum_{a} \frac{m_{a}}{M} \vec{r}_{a}, \\
\vec{\Pi} & =\sum_{a}\left[\vec{p}_{a}-e_{a} \vec{A}(\vec{R})\right]=\vec{P}-e \vec{A}(\vec{R}),
\end{aligned}
$$

where $M=\sum_{a} m_{a}$ and $e=\sum_{a} e_{a}$, and relative coordinates

$$
\begin{aligned}
\vec{x}_{a} & =\vec{r}_{a}-\vec{R}, \\
\vec{q}_{a} & =\vec{p}_{a}-\frac{m_{a}}{M} \vec{P},
\end{aligned}
$$


such that

$$
\begin{aligned}
{\left[x_{a}^{i}, q_{b}^{j}\right] } & =i \delta^{i j}\left(\delta_{a b}-\frac{m_{b}}{M}\right), \\
{\left[R^{i}, P^{j}\right] } & =i \delta^{i j}, \\
{\left[x_{a}^{i}, P^{j}\right] } & =\left[R^{i}, q_{a}^{j}\right]=0 .
\end{aligned}
$$

Next, we perform a canonical transformation $\phi$

$$
H^{\prime}=e^{-i \phi} H e^{i \phi}+\partial_{t} \phi,
$$

which assumes that the characteristic wavelength of the electromagnetic field is larger than the size of the system, as follows:

$$
\begin{aligned}
\phi & =\sum_{a} e_{a} \int_{0}^{1} d u \vec{x}_{a} \cdot \vec{A}\left(\vec{R}+u \vec{x}_{a}\right) \\
& =\sum_{a} e_{a}\left[x_{a}^{i} A^{i}+\frac{1}{2 !} x_{a}^{i} x_{a}^{j} A_{, j}^{i}+\ldots\right] .
\end{aligned}
$$

The scalar potential is transformed to

$$
\sum_{a} e_{a} A_{a}^{0}+\partial_{t} \phi=e A^{0}-D^{i} E^{i}-\frac{1}{2 !} D^{i j} E_{, j}^{i},
$$

where

$$
\begin{aligned}
D^{i} & =\sum_{a} e_{a} x_{a}^{i}, \\
D^{i j} & =\sum_{a} e_{a} x_{a}^{i} x_{a}^{j},
\end{aligned}
$$

and $A^{0} \equiv A^{0}(\vec{R}), \vec{E} \equiv \vec{E}(\vec{R})$, similarly $\vec{B} \equiv \vec{B}(\vec{R})$. The kinetic momentum is transformed to

$$
e^{-i \phi} \pi_{a}^{j} e^{i \phi}=\tilde{\pi}_{a}^{j}+\frac{m_{a}}{M} \Pi^{j},
$$

where

$$
\tilde{\pi}_{a}=\vec{q}_{a}+\frac{1}{2}\left(e_{a} \vec{x}_{a}+\frac{m_{a}}{M} \vec{D}\right) \times \vec{B},
$$

and the kinetic energy is

$$
e^{-i \phi} \sum_{a} \frac{\pi_{a}^{2}}{2 m_{a}} e^{i \phi}=\frac{\Pi^{2}}{2 M}+\frac{\vec{\Pi}}{M} \cdot \vec{D} \times \vec{B}+\sum_{a} \frac{\tilde{\pi}_{a}^{2}}{2 m_{a}} .
$$

Finally, the transformed Hamiltonian takes the form

$$
H^{\prime}=H_{B P}+H_{\partial E}+H_{\Pi},
$$

where

$$
\begin{aligned}
H_{B P}= & \sum_{a}\left\{\frac{\tilde{\pi}_{a}^{2}}{2 m_{a}}-\frac{\tilde{\pi}_{a}^{4}}{8 m_{a}^{3}}-\frac{e_{a}}{2 m_{a}} g_{a} \vec{s}_{a} \cdot \vec{B}+\frac{e_{a}}{8 m_{a}^{3}}\left[4 \tilde{\pi}_{a}^{2} \vec{s}_{a} \cdot \vec{B}+\left(g_{a}-2\right)\left\{\tilde{\pi}_{a} \cdot \vec{B}, \tilde{\pi}_{a} \cdot \vec{s}_{a}\right\}\right]\right. \\
& \left.-\frac{e_{a}\left(g_{a}-1\right)}{2 m_{a}^{2}} \vec{s}_{a} \times \vec{E} \cdot \tilde{\pi}_{a}\right\}+\sum_{a>b, b} \frac{e_{a} e_{b}}{4 \pi}\left\{\frac{1}{r_{a b}}-\frac{1}{2 m_{a} m_{b}} \tilde{\pi}_{a}^{i}\left(\frac{\delta^{i j}}{r_{a b}}+\frac{r_{a b}^{i} r_{a b}^{j}}{r_{a b}^{3}}\right) \tilde{\pi}_{b}^{j}\right. \\
& -\frac{2 \pi}{3}\left(r_{E a}^{2}+r_{E b}^{2}\right) \delta^{3}\left(r_{a b}\right)-\frac{2 \pi g_{a} g_{b}}{3 m_{a} m_{b}} \vec{s}_{a} \cdot \vec{s}_{b} \delta^{3}\left(r_{a b}\right)+\frac{g_{a} g_{b}}{4 m_{a} m_{b}} \frac{s_{a}^{i} s_{b}^{j}}{r_{a b}^{3}}\left(\delta^{i j}-3 \frac{r_{a b}^{i} r_{a b}^{j}}{r_{a b}^{2}}\right) \\
& +\frac{1}{2 r_{a b}^{3}}\left[\frac{g_{a}}{m_{a} m_{b}} \vec{s}_{a} \cdot \vec{r}_{a b} \times \tilde{\pi}_{b}-\frac{g_{b}}{m_{a} m_{b}} \vec{s}_{b} \cdot \vec{r}_{a b} \times \tilde{\pi}_{a}+\frac{\left(g_{b}-1\right)}{m_{b}^{2}} \vec{s}_{b} \cdot \vec{r}_{a b} \times \tilde{\pi}_{b}\right. \\
& \left.\left.\left.-\frac{\left(g_{a}-1\right)}{m_{a}^{2}} \vec{s}_{a} \cdot \vec{r}_{a b} \times \tilde{\pi}_{a}\right)\right]\right\}, \\
& H_{\partial E}=-\sum_{a} \frac{e_{a}}{6} r_{E a}^{2} \nabla \vec{E}-\frac{1}{2 !} D^{i j} E_{, j}^{i}-\sum_{a} \frac{e_{a}\left(g_{a}-1\right)}{4 m_{a}^{2}} \epsilon^{i k l}\left\{s_{a}^{i} E_{, j}^{k} x_{a}^{j}, \tilde{\pi}_{a}^{l}\right\}, \\
& H_{\Pi}=\frac{\vec{\Pi}^{2}}{2 M}+e A^{0}-\vec{D} \cdot \vec{E}+\vec{D} \cdot \vec{B} \times \frac{\vec{\Pi}}{M}-\frac{1}{8 M^{3}} \Pi^{4}-\frac{1}{2 M^{3}} \Pi^{2} \vec{\Pi} \cdot \vec{D} \times \vec{B} \\
& +\Pi^{i} Q_{i j} \Pi^{j}+\frac{1}{2}\left\{\Pi^{i}, Q_{i}\right\},
\end{aligned}
$$




$$
\begin{aligned}
Q^{i j}= & -\frac{\delta^{i j}}{2 M^{2}}\left(\sum_{a} \frac{\tilde{\pi}_{a}^{2}}{2 m_{a}}+\sum_{a>b, b} \frac{e_{a} e_{b}}{4 \pi r_{a b}}\right)-\frac{1}{2 M^{2}}\left(\sum_{a} \frac{\tilde{\pi}_{a}^{i} \tilde{\pi}_{a}^{j}}{m_{a}}+\sum_{a>b, b} \frac{e_{a} e_{b}}{4 \pi} \frac{r_{a b}^{i} r_{a b}^{j}}{r_{a b}^{3}}\right) \\
& +\sum_{a} \frac{e_{a}}{4 M^{2} m_{a}}\left[2 \delta^{i j} \vec{s}_{a} \cdot \vec{B}+\left(g_{a}-2\right) B^{i} s_{a}^{j}\right] \\
Q^{i}= & \sum_{a} \frac{e_{a}}{4 M m_{a}^{2}}\left[4 \tilde{\pi}_{a}^{i} \vec{s}_{a} \cdot \vec{B}+\left(g_{a}-2\right) B^{i} \tilde{\pi}_{a} \cdot \vec{s}_{a}+\left(g_{a}-2\right) s_{a}^{i} \tilde{\pi}_{a} \cdot \vec{B}\right] \\
& +\sum_{a>b, b} \frac{e_{a} e_{b}}{4 \pi}\left[\frac{1}{2 M m_{a} r_{a b}^{3}}\left(\vec{s}_{a} \times \vec{r}_{a b}\right)^{i}-\frac{1}{2 M m_{b}}\left(\frac{\delta^{i j}}{r_{a b}}+\frac{r_{a b}^{i} r_{a b}^{j}}{r_{a b}^{3}}\right) \tilde{\pi}_{b}^{j}\right] \\
& \quad-\sum_{a} \frac{e_{a}\left(g_{a}-1\right)}{2 m_{a} M}\left(\vec{s}_{a} \times \vec{E}\right)^{i}-\sum_{a} \frac{1}{4 M m_{a}^{2}}\left\{\tilde{\pi}_{a}^{2}, \tilde{\pi}_{a}^{i}\right\} .
\end{aligned}
$$

In order to simplify the derivation of the effective Hamiltonian $H_{\text {eff }}$ in Eq. (16), we perform the next canonical transformation $\phi$. In this case it should be noted that the $Q_{0}^{i}$ operator

$$
\begin{aligned}
Q_{0}^{i}= & \left.Q^{i}\right|_{\vec{E}=\vec{B}=0} \\
= & \frac{1}{2 M} \sum_{a \neq b, b} \frac{e_{a} e_{b}}{4 \pi}\left[\frac{1}{m_{a} r_{a b}^{3}}\left(\vec{s}_{a} \times \vec{r}_{a b}\right)^{i}\right. \\
& \left.-\frac{1}{m_{b}}\left(\frac{\delta^{i j}}{r_{a b}}+\frac{r_{a b}^{i} r_{a b}^{j}}{r_{a b}^{3}}\right) q_{b}^{j}\right]-\sum_{a} \frac{1}{2 M m_{a}^{2}} \vec{q}_{a}^{2} q_{a}^{i}
\end{aligned}
$$

can be expressed as a commutator

$$
\vec{Q}_{0}=i\left[H_{S}, \vec{T}\right]
$$

where

$$
\vec{T}=\frac{1}{2 M} \sum_{a}\left(\frac{\vec{s}_{a} \times \vec{q}_{a}}{m_{a}}-\frac{q_{a}^{j} \vec{x}_{a} q_{a}^{j}}{m_{a}}-\sum_{b \neq a} \frac{e_{a} e_{b}}{4 \pi} \frac{\vec{x}_{a}}{r_{a b}}\right)
$$

and $H_{S}$ is the nonrelativistic Hamiltonian of the bound system

$$
H_{S}=\sum_{a} \frac{\vec{q}_{a}^{2}}{2 m_{a}}+\sum_{a>b, b} \frac{e_{a} e_{b}}{4 \pi r_{a b}} .
$$

Consequently, we assume that

$$
\phi=-\vec{T} \cdot \vec{\Pi}
$$

and obtain a new Hamiltonian $H^{\prime \prime}$

$$
\begin{aligned}
H^{\prime \prime} & =e^{-i \phi} H^{\prime} e^{i \phi}+\partial_{t} \phi \\
& =H^{\prime}+\delta H,
\end{aligned}
$$

where

$$
\begin{aligned}
\delta H= & -i\left[H_{S}+\frac{\Pi^{2}}{2 M}+e A^{0}-\vec{D} \cdot\left(\vec{E}+\frac{\vec{\Pi}}{M} \times \vec{B}\right)\right. \\
& \left.-i \partial_{t}, \vec{T} \cdot \vec{\Pi}\right] .
\end{aligned}
$$

We will limit the effective Hamiltonian to terms that are independent, linear in electromagnetic field strength, and quadratic in electric field, thus neglecting the higher order terms in Eq. (16), so

$$
\begin{aligned}
H_{\mathrm{eff}}= & \left\langle\psi_{S}\left|H^{\prime \prime}-H_{B P}\right|_{\vec{E}=\vec{B}=0} \mid \psi_{S}\right\rangle \\
& +\left\langle\psi_{S}\left|H^{\prime \prime} \frac{1}{\left(\mathcal{E}_{S}-H_{S}\right)^{\prime}} H^{\prime \prime}\right| \psi_{S}\right\rangle
\end{aligned}
$$

The expectation value of $\delta H$ on $\psi_{S}$ is

$$
\begin{aligned}
\langle\delta H\rangle= & i\left\langle\left[\vec{D} \cdot\left(\vec{E}+\frac{\vec{\Pi}}{M} \times \vec{B}\right), \vec{T} \cdot \vec{\Pi}\right]\right\rangle \\
= & -\frac{\epsilon^{i j k}}{4 M}\left\{\left(\vec{E}+\frac{\vec{\Pi}}{M} \times \vec{B}\right)^{i}, \Pi^{j}\right\} \\
& \times \sum_{a}\left\langle\left(\frac{e_{a}}{m_{a}}-\frac{e}{M}\right)\left(s_{a}^{k}+l_{a}^{k}\right)\right\rangle \\
& -\frac{1}{6}\langle\vec{D} \cdot \vec{T}+\vec{T} \cdot \vec{D}\rangle \vec{\nabla} \cdot \vec{E}
\end{aligned}
$$

The resulting effective Hamiltonian from Eq. (48), after rearrangement, details of which are presented in the following sections, becomes

$$
\begin{aligned}
H_{\mathrm{eff}}= & e A^{0}+\frac{\Pi^{2}}{2 M}\left(1-\frac{\mathcal{E}_{S}}{M}\right)-\frac{\Pi^{4}}{8 M^{3}}-\frac{e}{6} R^{2} \nabla \cdot \vec{E}(50) \\
& -\frac{e}{2 M}(g+\delta g) \vec{S} \cdot \vec{B}+\frac{e}{2 M^{2}}(g-1) \vec{S} \cdot \vec{\Pi} \times \vec{E} \\
& +\frac{e}{2 M^{3}}\left[\Pi^{2} \vec{S} \cdot \vec{B}+\frac{(g-2)}{2} \vec{S} \cdot \vec{\Pi} \vec{B} \cdot \vec{\Pi}\right]-\frac{\alpha_{E}}{2} \vec{E}^{2}
\end{aligned}
$$

where $\vec{S}$ is a global spin operator, and formulas for $R^{2}, g, \delta g$, and $\alpha_{E}$ are presented in the following sections. 


\section{A. Mean square charge radius}

The mean square charge radius is defined as a coefficient at $\nabla \vec{E}$, see Eq. (50). It is present in $H_{\partial E}$

$$
H_{\partial E}=-\sum_{a} \frac{e_{a}}{6} \nabla \vec{E}\left[r_{E a}^{2}+x_{a}^{2}+\frac{\left(g_{a}-1\right)}{m_{a}^{2}} \vec{x}_{a} \times \vec{q}_{a} \cdot \vec{s}_{a}\right]
$$

and also in $\delta H$

$$
\langle\delta H\rangle=-\frac{1}{6} \nabla \vec{E}\langle\vec{T} \cdot \vec{D}+\vec{D} \cdot \vec{T}\rangle .
$$

For the total mean square charge radius $R^{2}$ of the system, we obtain

$e R^{2}=\sum_{a} e_{a}\left[r_{E a}^{2}+\left\langle x_{a}^{2}\right\rangle+\frac{\left(g_{a}-1\right)}{m_{a}^{2}}\left\langle\vec{x}_{a} \times \vec{q}_{a} \cdot \vec{s}_{a}\right\rangle+2\left\langle\vec{x}_{a} \cdot \vec{T}\right\rangle\right]$.

The first two terms are widely known, the third one was recently discovered by Flambaum et al. [6], and the last term is new. It would be interesting to calculate them for deuteron, for which the the charge radius is well known from atomic isotope shift measurements [7]. However, in the presence of strong interactions the formula for $R^{2}$ may change, and this should be verified using the effective chiral perturbation theory.

\section{B. Electric dipole polarizability}

The energy shift Eqs. (48) due to the electric dipole polarizability Eq. (50) is

$$
-\frac{\alpha_{E}}{2} \vec{E}^{2}=\left\langle\psi_{S}\left|(\vec{D}+\delta \vec{D}) \cdot \vec{E} \frac{1}{\mathcal{E}_{S}-H_{S}}(\vec{D}+\delta \vec{D}) \cdot \vec{E}\right| \psi_{S}\right\rangle
$$

so one obtains for $\alpha_{E}$

$$
\alpha_{E}=\frac{2}{3}\left\langle\psi_{S}\left|(\vec{D}+\delta \vec{D}) \frac{1}{H_{S}-\mathcal{E}_{S}}(\vec{D}+\delta \vec{D})\right| \psi_{S}\right\rangle .
$$

The relativistic correction to the electric dipole operator $\delta \vec{D}$ comes from Eq. (47)

$$
-\delta \vec{D} \cdot \vec{E}=-i\left[e A^{0}-i \partial_{t}, \vec{T} \cdot \vec{\Pi}\right]
$$

so

$$
\delta \vec{D}=e \vec{T}
$$

and $\vec{T}$ is defined in Eq. (43). In all the previous calculations of the electric dipole polarizability of nuclei, the contribution coming from $\delta D$ was missing. This correction is particularly important for muonic atoms, where nuclear polarizability effects are large.

\section{Kinetic energy}

When the electromagnetic field is neglected, the expectation value of $H^{\prime \prime}-\mathcal{E}_{S}$ is

$$
H_{\mathrm{eff}}=\frac{\Pi^{2}}{2 M}-\frac{\Pi^{4}}{8 M^{3}}+\Pi^{i} \Pi^{j}\left\langle Q_{0}^{i j}\right\rangle,
$$

where

$$
\begin{aligned}
Q_{0}^{i j}= & \left.Q^{i j}\right|_{\vec{E}=\vec{B}=0} \\
= & -\frac{\delta^{i j}}{2 M^{2}}\left(\sum_{a} \frac{\vec{q}_{a}^{2}}{2 m_{a}}+\sum_{a>b, b} \frac{e_{a} e_{b}}{4 \pi r_{a b}}\right) \\
& -\frac{1}{2 M^{2}}\left(\sum_{a} \frac{q_{a}^{i} q_{a}^{j}}{m_{a}}+\sum_{a>b, b} \frac{e_{a} e_{b}}{4 \pi} \frac{r_{a b}^{i} r_{a b}^{j}}{r_{a b}^{3}}\right)
\end{aligned}
$$

The expectation value of the second term vanishes, while that of the first term is $\mathcal{E}_{S}$, so

$$
\begin{aligned}
H_{\mathrm{eff}} & =\frac{\Pi^{2}}{2 M}\left(1-\frac{\mathcal{E}_{S}}{M}\right)-\frac{\Pi^{4}}{8 M^{3}} \\
& \approx \frac{\Pi^{2}}{2\left(M+\mathcal{E}_{S}\right)}-\frac{\Pi^{4}}{8\left(M+\mathcal{E}_{S}\right)^{3}} .
\end{aligned}
$$

$H_{\text {eff }}$ is a kinetic energy with the total mass being the sum of individual masses and the binding energy, as it should be. This is in agreement with Eq. (50).

\section{Spin in the external homogenous electric field}

Hereinafter we assume that the electric and the magnetic fields are homogenous. The magnetic moment $\mu$ of the compound system is defined as

$$
\vec{\mu}=\left\langle\sum_{a} \frac{e_{a}}{2 m_{a}}\left(\vec{l}_{a}+g_{a} \vec{s}_{a}\right)\right\rangle \equiv \frac{e}{2 M} g \vec{S}
$$

where the last equation defines the $g$-factor, and $\vec{l}_{a}=\vec{x}_{a} \times \vec{q}_{a}$ and $\vec{S}=\sum_{a}\left(\vec{l}_{a}+\vec{s}_{a}\right)$. The coupling of the static magnetic moment to the magnetic field is

$$
H_{\mathrm{eff}}=-\vec{\mu} \cdot \vec{B} .
$$

When the system moves, the magnetic moment couples to the electric field as follows

$$
H_{\mathrm{eff}}=\frac{\vec{\Pi} \times \vec{E}}{2 M} \cdot \sum_{a}\left\langle\frac{e_{a}\left(g_{a}-1\right)}{m_{a}} \vec{s}_{a}\right\rangle+\delta H,
$$

where

$$
\delta H=\frac{\vec{\Pi} \times \vec{E}}{2 M} \cdot \sum_{a}\left\langle\left(\frac{e_{a}}{m_{a}}-\frac{e}{M}\right)\left(\vec{s}_{a}+\vec{l}_{a}\right)\right\rangle .
$$


After combining both terms

$$
\begin{aligned}
H_{\mathrm{eff}} & =\frac{\vec{\Pi} \times \vec{E}}{2 M} \cdot \sum_{a}\left\langle\frac{e_{a}}{m_{a}}\left(g_{a} \vec{s}_{a}+\vec{l}_{a}\right)-\frac{e}{M}\left(\vec{l}_{a}+\vec{s}_{a}\right)\right\rangle \\
& =\frac{e}{2 M^{2}}(g-1) \vec{S} \cdot \vec{\Pi} \times \vec{E}
\end{aligned}
$$

the coupling of the moving spin to the electric field coincides with that in Eq. (50).

\section{E. Spin in the external homogenous magnetic field}

Corrections of order $O\left(\vec{\Pi}^{2}\right)$ to the coupling of the spin to the magnetic field are

$$
H_{\mathrm{eff}}=\Pi^{i}\left\langle Q_{B}^{i j}\right\rangle \Pi^{j}+\delta H,
$$

where the part of $Q^{i j}$ that is linear in $\vec{B}$ is

$$
\begin{aligned}
\left\langle Q_{B}^{i j}\right\rangle= & \frac{1}{2 M^{2}} \sum_{a} \frac{e_{a}}{2 m_{a}}\left\langle\delta^{i j} \vec{x}_{a} \times \vec{q}_{a} \cdot \vec{B}-\left(\vec{x}_{a} \times \vec{B}\right)^{i} q_{a}^{j}\right. \\
& \left.-q_{a}^{i}\left(\vec{x}_{a} \times \vec{B}\right)^{j}+\left(2 \delta^{i j} \vec{s}_{a} \cdot \vec{B}+\left(g_{a}-2\right) B^{i} s_{a}^{j}\right)\right\rangle \\
= & \frac{1}{2 M^{2}} \sum_{a} \frac{e_{a}}{2 m_{a}}\left\langle 2 \delta^{i j}\left(\vec{l}_{a}+\vec{s}_{a}\right) \cdot \vec{B}-B^{i} l_{a}^{j}\right. \\
& \left.+\left(g_{a}-2\right) B^{i} s_{a}^{j}\right\rangle,
\end{aligned}
$$

where we used the expectation value identity

$$
\left\langle x_{a}^{i} q_{a}^{j}\right\rangle=\frac{1}{2}\left\langle x_{a}^{i} q_{a}^{j}-x_{a}^{j} q_{a}^{i}\right\rangle .
$$

The contribution from the additional canonical transformation Eq. (49) is

$$
\delta H=-\frac{\vec{\Pi} \times \vec{B}}{2 M^{2}} \sum_{a}\left\langle\left(\frac{e_{a}}{m_{a}}-\frac{e}{M}\right) \vec{\Pi} \times\left(\vec{s}_{a}+\vec{l}_{a}\right)\right\rangle
$$

The total $O\left(\vec{\Pi}^{2}\right)$ interaction takes the form

$$
H_{\mathrm{eff}}=\frac{e}{2 M^{3}}\left[\Pi^{2} \vec{S} \cdot \vec{B}+\frac{(g-2)}{2} \vec{S} \cdot \vec{\Pi} \vec{B} \cdot \vec{\Pi}\right]
$$

and coincides with that in Eq. (50).

\section{MAGNETIC MOMENT}

The relativistic corrections to the magnetic moment of bound states with arbitrary particle masses have already been considered in the literature [1, 3, 8] and very recently in [10]. Here we rederive the general formula for the arbitrary state, obtain the known result for the magnetic moment of hydrogen-like ions in the $\mathrm{S}$ state, and confirm and obtain a more accurate result for positronium ion $\mathrm{Ps}^{-}$in the ground state. Consider the relativistic interaction with the magnetic field resulting from $H_{B P}$ in Eq. (36), and neglect the terms quadratic in $\vec{B}$.

$$
\begin{aligned}
\delta H= & -\sum_{a} \frac{e_{a}}{2 m_{a}} g_{a} \vec{s}_{a} \cdot \vec{B}+\sum_{a} \frac{1}{4 m_{a}^{3}}\left[q_{a}^{2} \vec{D}_{a} \times \vec{q}_{a} \cdot \vec{B}+2 e_{a} q_{a}^{2} \vec{s}_{a} \cdot \vec{B}+e_{a}\left(g_{a}-2\right) \vec{q}_{a} \cdot \vec{s}_{a} \vec{q}_{a} \cdot \vec{B}\right] \\
& +\sum_{a \neq b, b} \frac{e_{a} e_{b}}{4 \pi}\left[-\frac{1}{4 m_{a} m_{b}} q_{a}^{i}\left(\frac{\delta^{i j}}{r_{a b}}+\frac{r_{a b}^{i} r_{a b}^{j}}{r_{a b}^{3}}\right)\left(\vec{D}_{b} \times \vec{B}\right)^{j}\right. \\
& \left.+\frac{1}{4 r_{a b}^{3}} \frac{g_{a}}{m_{a} m_{b}}\left(\vec{s}_{a} \times \vec{r}_{a b}\right) \cdot\left(\vec{D}_{b} \times \vec{B}\right)-\frac{1}{4 r_{a b}^{3}} \frac{\left(g_{a}-1\right)}{m_{a}^{2}}\left(\vec{s}_{a} \times \vec{r}_{a b}\right) \cdot\left(\vec{D}_{a} \times \vec{B}\right)\right],
\end{aligned}
$$

where

$$
\vec{D}_{a}=e_{a} \vec{x}_{a}+\frac{m_{a}}{M} \vec{D}
$$

Eq. (71) agrees with the former result of Hegstrom [1], and is essentially the same as that in Ref. [3]. For S-states, only the spin dependent terms in (71) contribute, so

$$
\begin{aligned}
\delta H= & -\sum_{a} \frac{e_{a}}{2 m_{a}} \vec{s}_{a} \cdot \vec{B}\left\{g_{a}-\frac{\vec{q}_{a}^{2}}{m_{a}^{2}}\left(\frac{2}{3}+\frac{g_{a}}{6}\right)\right. \\
& \left.+\frac{1}{3} \sum_{b \neq a} \frac{e_{b}}{4 \pi} \frac{\vec{r}_{a b}}{r_{a b}^{3}} \cdot\left[\frac{g_{a}}{m_{b}} \vec{D}_{b}-\frac{g_{a}-1}{m_{a}} \vec{D}_{a}\right]\right\} .
\end{aligned}
$$

First we will consider the hydrogen-like ion. It is a system consisting of one electron of mass $m$ and charge $-e$, and the nucleus of charge $Z e$ and mass $m_{N}$. We neglect the spin of 
the nucleus, so the Hamiltonian is

$$
\begin{aligned}
\delta H= & \frac{e}{2 m} \vec{s} \cdot \vec{B}\left\{g_{e}-\frac{\vec{q}^{2}}{m^{2}}\left(\frac{2}{3}+\frac{g_{e}}{6}\right)+\frac{Z \alpha}{3 M r_{e N}}\right. \\
& \times\left[g_{e}\left(-Z \frac{M}{m_{N}}+(Z-1) \frac{m_{N}}{M}\right)\right. \\
& \left.\left.+\left(g_{e}-1\right)\left(\frac{M}{m}+(Z-1) \frac{m}{M}\right)\right]\right\} .
\end{aligned}
$$

The correction $\delta g$ is given by the expectation value of the Hamiltonian on the hydrogen-like system state $\phi=\phi_{n l m_{l}}$, where $n, l, m_{l}$ are respective quantum numbers

$$
\langle\delta H\rangle=\frac{e}{2 m} \vec{s} \cdot \vec{B}\left(g_{e}+\delta g\right),
$$

where

$$
\begin{aligned}
\delta g= & -\frac{(Z \alpha)^{2}}{3 n^{2}(1+x)^{2}}\left[-\frac{g_{e}}{2}+4-\frac{1}{1+x}\right. \\
& \left.+Z x^{2}\left(g_{e}+\frac{1}{1+x}\right)\right]
\end{aligned}
$$

and $x=m / m_{N}$, in agreement with Refs. [3, 8].

In the case of the positronium ion $\mathrm{Ps}^{-}$in the ground state, the spin comes only from the positron, since two electrons are in the singlet state. Hence from the beginning we neglect terms proportional to electron spin, and assume that indices 1,2 refer to electrons, while index 3 refers to the positron.

$$
\begin{aligned}
\delta H= & -\frac{e}{2 m} \vec{s}_{3} \cdot \vec{B}\left\{g_{e}-\frac{\vec{q}_{3}^{2}}{m^{2}}\left(\frac{2}{3}+\frac{g_{e}}{6}\right)\right. \\
& -\frac{\alpha}{9 m}\left[\frac{5}{3}\left(\frac{1}{r_{13}}+\frac{\vec{r}_{13} \cdot \vec{r}_{23}}{r_{13}^{3}}\right)+g_{e} \frac{\vec{r}_{13} \cdot\left(\vec{r}_{12}-\vec{r}_{23}\right)}{r_{13}^{3}}\right. \\
& \left.\left.+\frac{5}{3}\left(\frac{1}{r_{23}}+\frac{\vec{r}_{13} \cdot \vec{r}_{23}}{r_{23}^{3}}\right)-g_{e} \frac{\vec{r}_{23} \cdot\left(\vec{r}_{12}+\vec{r}_{13}\right)}{r_{23}^{3}}\right]\right\}
\end{aligned}
$$

As previously, we find a correction to the magnetic moment of $\mathrm{Ps}^{-}$from the expectation value of the Hamiltonian (77).

$$
\langle\delta H\rangle=-\frac{e}{2 m} \vec{s}_{3} \cdot \vec{B} g_{\mathrm{Ps}^{-}} .
$$

Of note, $g_{\mathrm{Ps}^{-}}$is defined differently from the g-factor in Eq. (61). Following this definition and Eq. (77) $g_{\mathrm{Ps}^{-}}$is

$$
\begin{aligned}
g_{\mathrm{Ps}^{-}}= & g_{e}+\alpha^{2} \delta g, \\
\delta g= & -\left\langle\vec{p}_{3}^{2}\left(\frac{2}{3}+\frac{g_{e}}{6}\right)+\frac{1}{r_{13}}\left(\frac{10}{27}+\frac{2}{9} g_{e}\right)\right. \\
& \left.+\frac{\vec{r}_{13} \cdot \vec{r}_{23}}{r_{13}^{3}}\left(\frac{10}{27}-\frac{4}{9} g_{e}\right)\right\rangle,
\end{aligned}
$$

where for convenience we used in the last equation atomic units, so above matrix elements are dimensionless. Table I presents expectation value of operators in the ground state of $\mathrm{Ps}^{-}$calculated numerically, where the uncertainty for our total g-factor $g_{\mathrm{Ps}^{-}}$is estimated by $2 \alpha^{4} \delta g$. Our value for $\delta g$ is in agreement with the one obtained in [10] $-0.51(1)$, but is significantly more accurate and includes the leading QED effects. Surprisingly we do not agree with the corresponding formula obtained in [10], which is much different and not equivalent to that of ours in Eq. (80), and also we disagree with their total $\mathrm{g}$-factor $g_{\mathrm{Ps}^{-}}=2.00461(1)$.

TABLE I: Expectation values of operators in 80 on the ground state of $\mathrm{Ps}^{-}$in atomic units, fundamental constants are from Ref. [9]

\begin{tabular}{lc}
\hline \hline Energy & -0.262005070 \\
$\left\langle\vec{p}_{3}^{2}\right\rangle$ & 0.257532962 \\
$\left\langle\frac{1}{r_{13}}\right\rangle$ & 0.339821023 \\
$\left\langle\frac{\vec{r}_{13} \cdot \vec{r}_{23}}{r_{13}^{3}}\right\rangle$ & 0.046478421 \\
$\delta g$ & $-0.510551028(1)$ \\
$g_{e}$ & 2.002319304 \\
$g_{\mathrm{Ps}^{-}}$ & $2.002292117(3)$ \\
\hline
\end{tabular}

\section{SUMMARY}

We have presented an approach to derive an effective Hamiltonian that governs the dynamics of the whole bound system from individual Hamiltonians of its ingredients, including leading relativistic corrections. This approach is based on our two former works [2, 3], and in comparison to them it is much simpler. We derived a formula for the charge radius, which can be used for systems such as nuclei. Besides the known terms, it includes new terms which until now have not been taken into account in the calculation of nuclear charge radii. Similarly, the electric dipole polarizability includes corrections to the electric dipole moment $\delta D$ that have been omitted in all previous calculations of the nuclear polarizability. The obtained formula for the magnetic moment is in agreement with that obtained previously [1, 3, 8] and we improve the result published recently for the positronium ion [10]. The presented approach can also be used for nuclei, to calculate their electromagnetic moment, but this requires incorporation of strong interactions via the chiral perturbation theory. It is especially important in view of very accurate results for nuclear charge radii differences between isotopes obtained from atomic spectroscopy [7, 11].

\section{Acknowledgments}

Authors would like to acknowledge support by NCN grant 2012/04/A/ST2/00105. 
[3] K. Pachucki, Phys. Rev. A 78, 012504 (2008).

[4] H.A. Bethe and E.E. Salpeter, Quantum Mechanics of One-and Two-Electron Atoms (Academic, New York, 1957).

[5] K. Pachucki, Phys. Rev. A 69, 052502 (2004).

[6] A. Ong, J. C. Berengut and V. V. Flambaum, Phys. Rev. C 82, 014320 (2010).

[7] C. G. Parthey, A. Matveev, J. Alnis, R. Pohl, T. Udem, U. D. Jentschura, N. Kolachevsky, and T. W. Hänsch, Phys. Rev. Lett. 104, 233001 (2010).
[8] M. I. Eides and T. J. S. Martin, Phys. Rev. Lett. 105, 100402 (2010).

[9] P. J. Mohr, B. N. Taylor, and D. B. Newell, Rev. Mod. Phys. 84, 1527 (2012)

[10] Y. Liang, P. L. McGrath and A. Czarnecki, New J. Phys. 16, 063045 (2014).

[11] Z.-T. Lu, P. Mueller, G. W. F. Drake, W. Nörtershäuser, Steven C. Pieper, and Z.-C. Yan, Rev. Mod. Phys. 85, 1383 (2013). 